\title{
Optimization of the Vibro-Impact Capsule System for Promoting Progression Speed
}

\author{
Liao Maolin ${ }^{1, \star}$, Yan Yao ${ }^{2}$, and Liu Yang ${ }^{3}$ \\ ${ }^{1}$ CNPC Drilling Research Institute, Beijing, 102206, China \\ ${ }^{2}$ University of Electronic Science and Technology of China, Chengdu, 611731, China \\ ${ }^{3}$ College of Engineering, Mathematics and Physical Sciences, University of Exeter, Rennes Drive, Exeter, EX4 4RN, UK
}

\begin{abstract}
This paper studies the dynamics of vibro-impact capsule systems with one-sided and double-sided constraints under variations of control parameters, including frequency of excitation, mass ratio, and stiffness ratio. The aim of this study is to optimize the progression speed of the capsule system. Extensive comparisons reveal that the capsule system with one-sided constraint is better than the one with double-sided constraints in terms of progression speed. Moreover, the system's period-one one-right-impact motion is proved as the ideal vibration condition due to its lowest energy consumption for impacts. According to the dynamic analyses of control parameters, an inner mass with its weight similar as the weight of capsule and a right constraint with a relative weak stiffness are beneficial for further accelerating the capsule system forwards.
\end{abstract}

\section{Introduction}

Pipelines play an important role in a large number of modern industries, which are essential for oil and gas transport, water supplies, and so on. With many pipelines being located in remote and harsh locations, such as underground or seabed, access for inspection, maintenance and repair work could be extremely difficult. Moreover, it becomes a costly issue if the pipeline has to be drained and production stopped while repair work takes place. Therefore, pipeline inspection devices capable of moving independently, with or against product flow would yield significant advantages over traditional pressure driven inspection tool in certain situations. In recent years, investigation of such a selfpropelled mechanism moving rectilinearly under internal vibration force when overcoming medium resistance has attracted great attention from researchers, e.g. [1-5]. The principle of such mechanism is that the rectilinear motion can be obtained by overcoming external resistance described as dry friction using an additional internal mass interacting with the main body of the system.

The dynamics of the vibro-impact capsule system, which consists of a capsule main body interacting with a harmonically driven internal mass, has been studied extensively by Liu et al. [5-11]. In [5], the model of the vibro-impact capsule system was firstly studied to provide a fundamental understanding of its dynamics. Dynamics of the system in various environments was investigated in [6], and numerical results show that the behaviour of the system becomes very complex when the capsule is moving in a fluid, but the nature of the friction mechanism becomes less significant when the weight of the internal mass

\footnotetext{
^e-mail: liaomldr@cnpc.com.cn
}

is smaller than the weight of the capsule. In [7], the nonlinear dynamics analysis has been conducted to identify the optimal amplitude and frequency of the applied force to achieve the required motion and the maximal speed. In [10], Páez Chávez et al. studied two practical problems for the capsule system, which were maximizing the rate of progression and directional control of the system by following a typical period-1 trajectory by means of pathfollowing techniques. However, the above studies were based on the dynamics of the capsule system with onesided constraint, and the performance of the system with double-sided constraints has not been investigated. Thus, it is reasonable to carry out a comparative study of vibroimpact capsule systems with one-sided and double-sided constraints in this paper, which can provide a better insight for design of such system with consideration of the optimum rate of progression.

The rest of this paper is organized as follows. In Section 2, mathematical modelling of the vibro-impact capsule systems with one-sided and double-sided constraints is studied. In Section 3, a comparative study of both the capsule systems through varying control parameters to explore the optimum rate of progression are conducted. Finally, some concluding remarks are drawn in Section 4.

\section{Mathematical Modelling}

Consider a two degrees-of-freedom system as shown in Fig. 1(a), which is composed of a movable internal mass $m_{1}$ interacting with a rigid capsule $m_{2}$ via a primary linear spring with stiffness $k$ and a viscous damper with damping coefficient $c$. The internal mass is driven by an external harmonic force with amplitude $P_{d}$ and frequency $\Omega$. On 
the right of the internal mass, a weightless plate is connected to the capsule by a linear spring with stiffness $k_{1}$, and a secondary weightless plate is connected to the capsule by a linear spring with stiffness $k_{2}$ on the left of the internal mass. Here, $X_{1}$ and $X_{2}$ represent the absolute displacements of the internal mass and the capsule, respectively. The internal mass will contact with the right plate when the relative displacement $X_{1}-X_{2}$ is larger or equals to the gap $G_{1}$, or contact with the left plate when the relative displacement $X_{2}-X_{1}$ is larger or equals to the gap $G_{2}$. When the left spring is removed (i.e. $k_{2}=0$ ) as shown in Fig. 1(b).

Introduce nondimensional parameters

$$
\begin{gathered}
\Omega_{0}=\sqrt{\frac{k}{m_{1}}}, \quad \omega=\frac{\Omega}{\Omega_{0}}, \quad \alpha=\frac{P_{d}}{P_{f}}, \\
\zeta=\frac{c}{2 m_{1} \Omega_{0}}, \quad \gamma=\frac{m_{2}}{m_{1}}, g_{1}=\frac{k}{P_{f}} G_{1}, \\
g_{2}=\frac{k}{P_{f}} G_{2}, \quad \beta_{1}=\frac{k_{1}}{k}, \quad \beta_{2}=\frac{k_{2}}{k},
\end{gathered}
$$

and nondimensional variables

$$
\begin{gathered}
\tau=\Omega_{0} t, \quad x_{1}=\frac{k}{P_{f}} X_{1}, \quad x_{2}=\frac{k}{P_{f}} X_{2}, \\
v_{1}=\frac{\mathrm{d} x_{1}}{\mathrm{~d} \tau}=\frac{k}{\Omega_{0} P_{f}} \dot{X}_{1}, \quad v_{2}=\frac{\mathrm{d} x_{2}}{\mathrm{~d} \tau}=\frac{k}{\Omega_{0} P_{f}} \dot{X}_{2} .
\end{gathered}
$$

The nondimensional equations of motion can be written as [12]

$$
\begin{aligned}
& \dot{x}_{1}(\tau)=v_{1}(\tau), \\
& \dot{v}_{1}(\tau)=\alpha \cos (\omega \tau)+f_{i}, \\
& \dot{x}_{2}(\tau)=v_{2}(\tau), \\
& \dot{v}_{2}(\tau)=\frac{1}{\gamma}\left(f_{i}+\left(1-\delta_{v}\right) S_{v}+\delta_{v} H_{f} S_{f}+\delta_{v}\left(1-H_{f}\right) f_{i}\right),
\end{aligned}
$$

where

$$
\begin{aligned}
f_{i}= & -2 \zeta\left(v_{1}(\tau)-v_{2}(\tau)\right)-\left(x_{1}(\tau)-x_{2}(\tau)\right) \\
& -H_{1} \beta_{1}\left(x_{1}(\tau)-x_{2}(\tau)-g_{1}\right) \\
& -H_{2} \beta_{2}\left(x_{1}(\tau)-x_{2}(\tau)+g_{2}\right) \\
H_{1}= & H\left(x_{1}(\tau)-x_{2}(\tau)-g_{1}\right) \\
H_{2}= & H\left(x_{2}(\tau)-x_{1}(\tau)-g_{2}\right) \\
H_{f}= & H\left(\left|f_{i}\right|-1\right) \\
\delta_{v}= & \delta\left(v_{2}(\tau)\right) \\
S_{v}= & \operatorname{sign}\left(v_{2}(\tau)\right) \\
S_{f}= & \operatorname{sign}\left(f_{i}\right) .
\end{aligned}
$$

\section{Bifurcation analysis}

In order to compare the system dynamics and optimize the progression speed of capsule, bifurcation analyses were carried out for both the capsule systems with one-sided and double-sided constraints by sweeping control parameters, including frequency of excitation, mass ratio, and stiffness ratio. The simulations were run for 200 cycles of the external excitation, and the data for the first 180 cycles were omitted to ensure the steady state responses, where the last 20 cycles were used to plot bifurcation diagrams, phase portraits, and time series.

\subsection{Frequency of excitation}

To investigate the influence of excitation frequency $\omega$ on the capsule progression, numerical simulations are carried out for $\omega \in[0.5,2.0]$, and the simulation results are presented in Fig. 2. According to the comparison of subplots (a) and (b), the dynamic responses of the capsule system with one-sided constraint are mainly periodic; while the chaotic motion is observed for the capsule system with two-sided constraints when the excitation frequency is low. Subplots (c), (d) show the progressions per unit time and per unit power for the capsule system with one-sided constraint, respectively. Both of their optimum progressions are achieved at $\omega=1.005$ where a period-one one-right-impact motion is detected. Subplots (e), (f) show the corresponding results for the capsule system with double-sided constraint, whose maximal progression speed is about $1 / 3$ of that for the capsule system with one-sided constraint.

\subsection{Mass ratio}

The effects of mass ratio $\gamma$ on the dynamic responses of both the capsule systems are studied via sweeping $\gamma \in$ $[1,10]$, and the obtained numerical results are shown in Fig. 3. One can observe from subplots (a) and (b) that, both the capsule systems experience a period-doubling and a reverse period doubling bifurcations as the increase of mass ratio. For the capsule system with double-sided constraints, the negative capsule progression lasts, see subplots (e) and (f); namely, the backward impacts dominate the capsule movement. As a comparison, the capsule system with one-sided constraint maintains forward progression with the progression speed deceasing when the mass ratio increases, see subplots (c) and (d). This observation indicates that a tiny inner mass is hard to vibrate a huge capsule; therefore, it is better to design the weight of the inner mass closing to that of the capsule.

\subsection{Stiffness ratio}

The dynamic responses of the capsule systems under varying the right stiffness ratio, $\beta_{1} \in[2,20]$, are investigated, and the obtained numerical results are presented in Fig. 4. As can be seen from subplots (c) and (d), the fastest progression speed of the capsule system with one-sided constraint is achieved at $\beta_{1}=6.05$ where a period-doubling bifurcation is observed. Similarly, the fastest progression speed of the capsule system with double-sided constraint is achieved at $\beta_{1}=4.10$ where a grazing bifurcation is detected, see subplots (e) and (f). Before appearance of the bifurcations, period-one motions are observed, and the progression speed keeps increasing as the increase of stiffness ratio, and then it drops quickly when passing the bifurcations. In addition, compare the two capsule systems 
with the same right stiffness ratio, the capsule system with one-sided constraint moves forwards faster.

\section{Concluding Remarks}

Optimization of vibro-impact capsule system to promote progression speed was carried out in this paper. Different capsule structures were compared and a series of control parameters were analyzed. Extensive comparisons revealed that the capsule system with one-sided constraint was better than that with double-sided constraints in terms of progression speed; moreover, the system's period-one one-right-impact motion was proved as the ideal vibration condition due to its lowest energy consumption for impacts. Meanwhile, according to the dynamic analyses of control parameters, an inner mass with its weight closing to the weight of capsule and a right constraint with the relative weak stiffness were benefit for further accelerating the capsule system forwards.

\section{Acknowledgements}

This research is funded by National Basic Research Program of China (2015CB251206), CNPC Scientific Research and Technology Development Program, and China Postdoctoral Science Foundation.

\section{References}

[1] F.L. Chernousko, J. Appl. Maths Mechs. 66, 1 (2002)

[2] N.N. Bolotnik, I.M. Zeidis, K. Zimmermann, S.F. Yatsun, J. Computer and Systems Sciences International 45, 157 (2006)

[3] H.B. Fang, J. Xu, J. Vibration and Control 17, 19 (2011)

[4] Z. Nagy, R.I. Leine, D.R. Frutiger, C. Glocker, B.J. Nelson, IEEE Trans. Robotics 28, 1058 (2012)

[5] Y. Liu, M. Wiercigroch, E. Pavlovskaia, H. Yu, Int J Mechanical Sciences 66, 2 (2013)

[6] Y. Liu, E. Pavlovskaia, M. Wiercigroch, Int J Mechanical Sciences 72, 39 (2013)

[7] Y. Liu, M. Wiercigroch, E. Pavlovskaia, Z.K. Peng, Int. J. Non-Linear Mech. 70, 30 (2015)

[8] Y. Liu, E. Pavlovskaia, M. Wiercigroch, Nonlinear Dyn. 83, 1029 (2016)

[9] Y. Liu, S. Islam, E. Pavlovskaia, M. Wiercigroch, Strojniški vestnik - Journal of Mechanical Engineering 62, 430 (2016)

[10] J. Páez Cháez, Y. Liu, E. Pavlovskaia, M. Wiercigroch, Communications in Nonlinear Science and Numerical Simulation 37, 102 (2016)

[11] Y. Liu, J. Páez Cháez, Nonlinear Dyn. 88, 1289 (2017)

[12] Y. Yan, Y. Liu, M. Liao, Nonlinear Dyn. 89, 1063 (2017) 
(a)

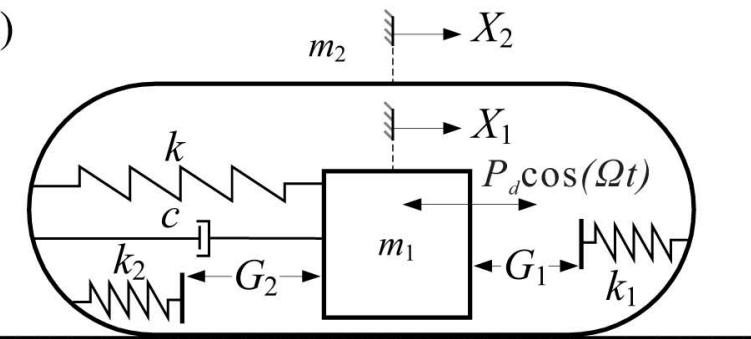

(b)

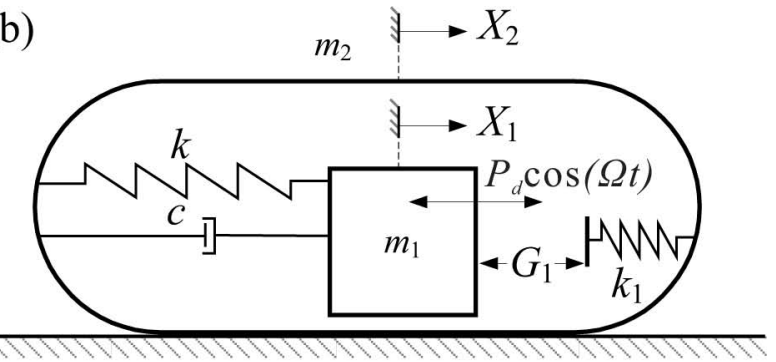

(c)
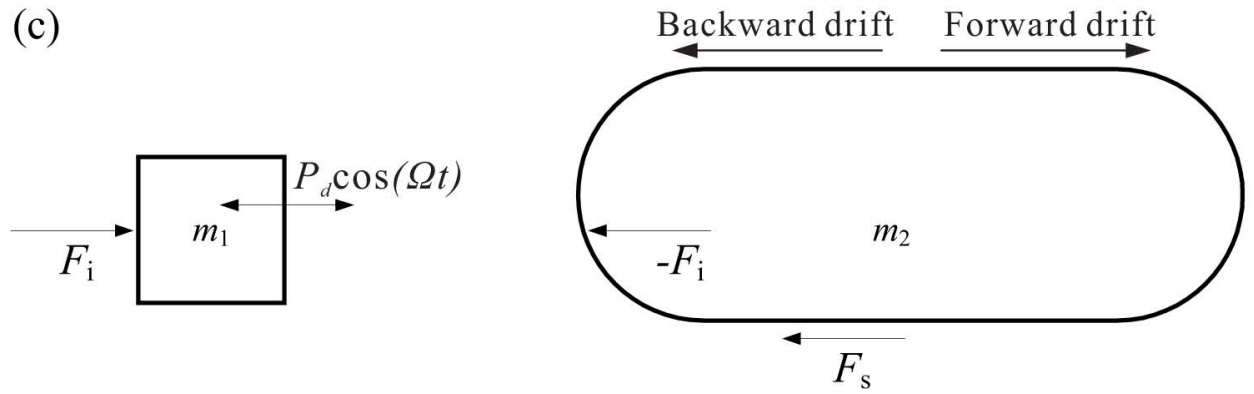

Figure 1. Physical models of the vibro-impact capsule systems, with (a) double-sided constraint, and (b) one-sided constraint. (c) Free body diagrams of the inner mass $m_{1}$ and the capsule $m_{2}$ [12].

(a)

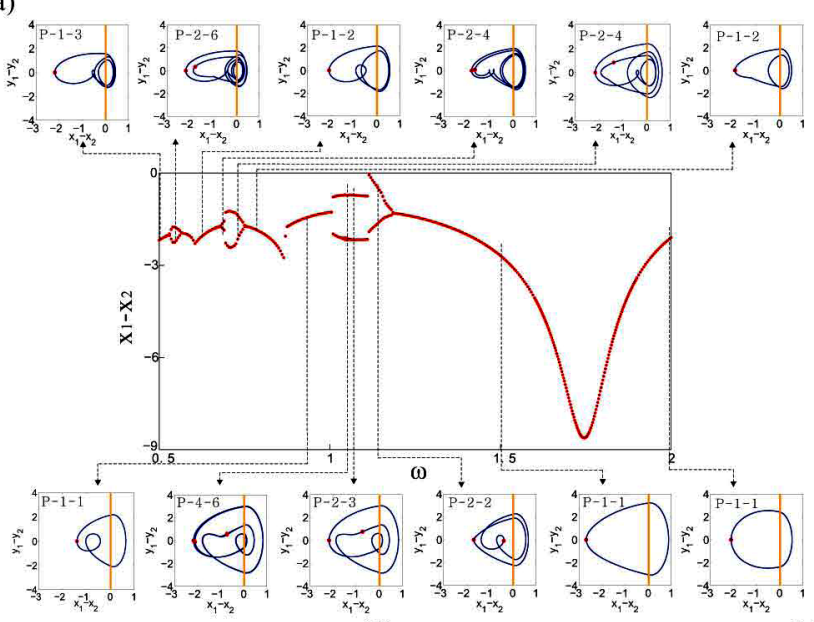

(c)

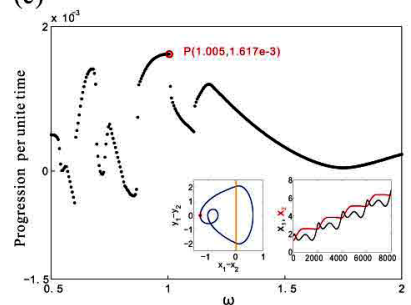

(d)

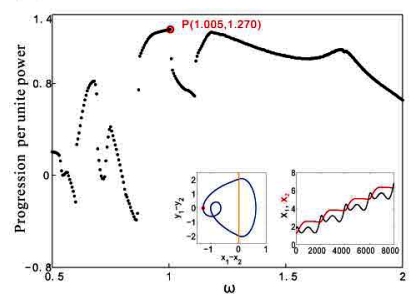

(b)

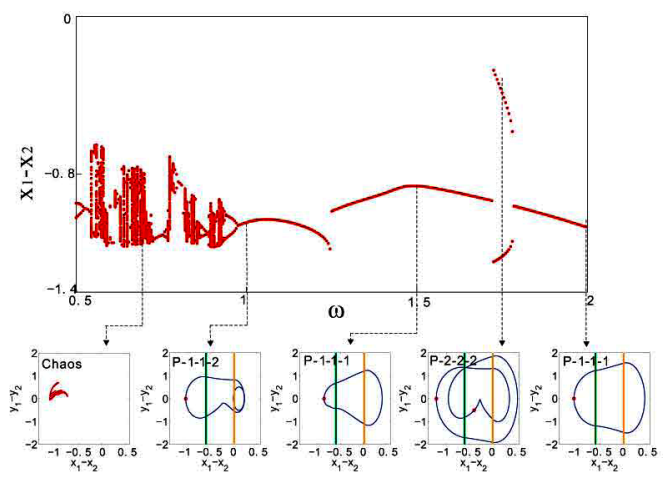

(f)

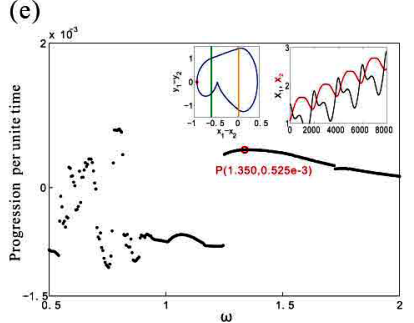

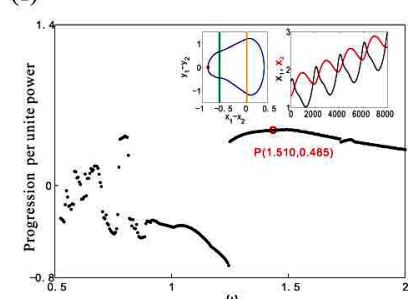

Figure 2. Bifurcation analyses of excitation frequency for the capsule systems with one-sided (a) and double-sided (b) constraints. The nondimensional parameters are $\omega \in[0.5,2.0], \xi=0.05, \gamma=4, a=1.6, \beta_{1}=12, g_{1}=0.02, \beta_{2}=5, g_{2}=-0.06$. The labels shown as $\mathrm{P}-\mathrm{n}-\mathrm{m}-1$ indicate that the trajectory is a period-n response with $\mathrm{m}$ left impacts and 1 right impacts. The vertical green and orange lines in panels represent the impact boundaries $x_{1}-x_{2}=g_{2}$ and $x_{1}-x_{2}=g_{1}$, respectively. Subplots (c), (d) show the progressions per unit time and per unit power for the capsule system with one-sided constraint, respectively. Subplots (e), (f) show the progressions for the capsule system with double-sided constraint. The position $P$ marked by a red circle indicates the obtained optimum progression, its corresponding phase portrait and time series of displacements for the inner mass (black) and the capsule (red) are displayed. 


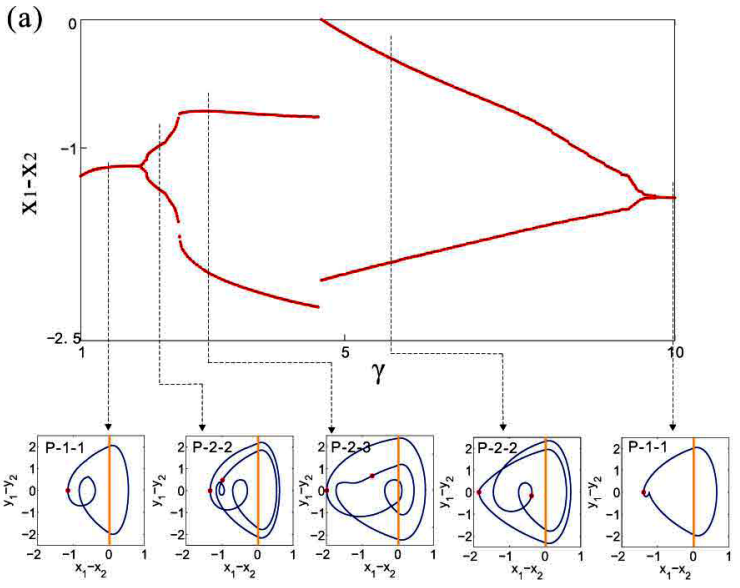

(c)

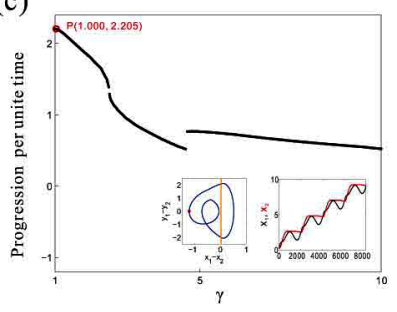

(d) $\times 10^{\circ}$

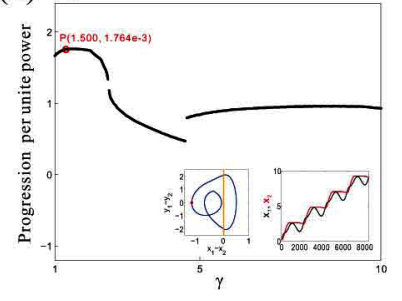

(b)

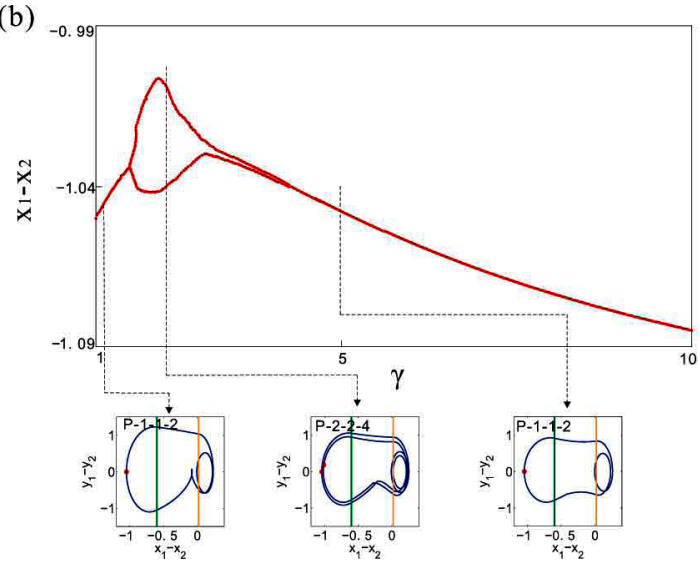

(e)

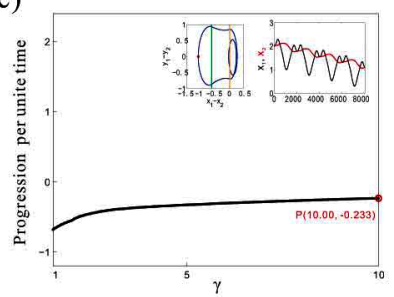

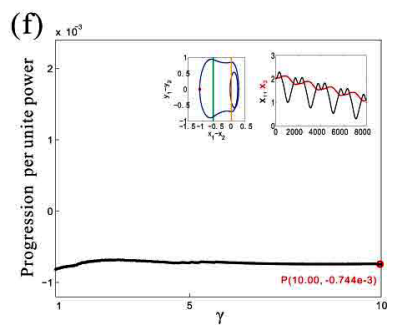

Figure 3. Bifurcation analyses of mass ratio for the capsule systems with one-sided (a) and double-sided (b) constraints. The nondimensional parameters are $\gamma \in[1,10], a=1.6, \omega=1.1, \xi=0.05, \beta_{1}=12, g_{1}=0.02, \beta_{2}=5, g_{2}=-0.06$. Subplots (c), (d) show the progressions per unit time and per unit power for the capsule system with one-sided constraint. Subplots (e), (f) show the progressions for the capsule system with double-sided constraint.

(a)

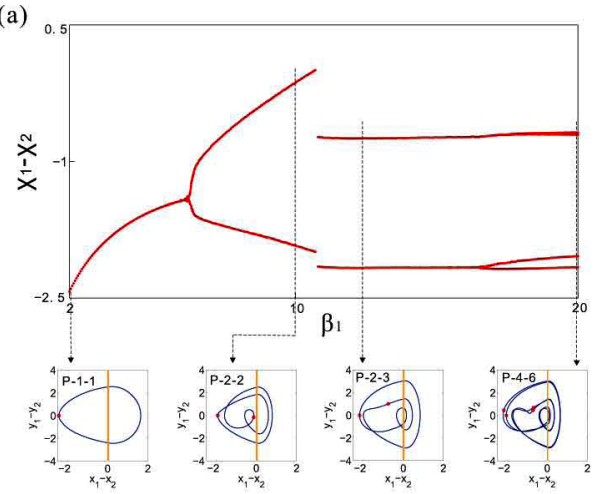

(c)

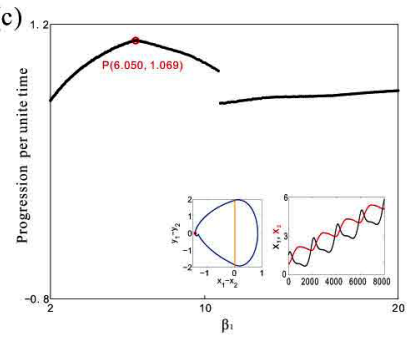

(d)

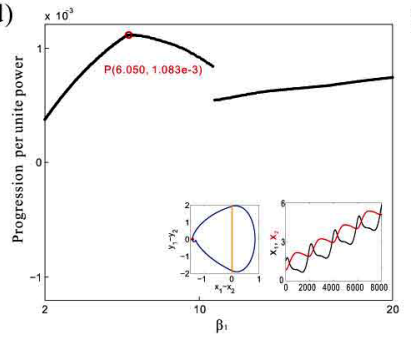

(b)

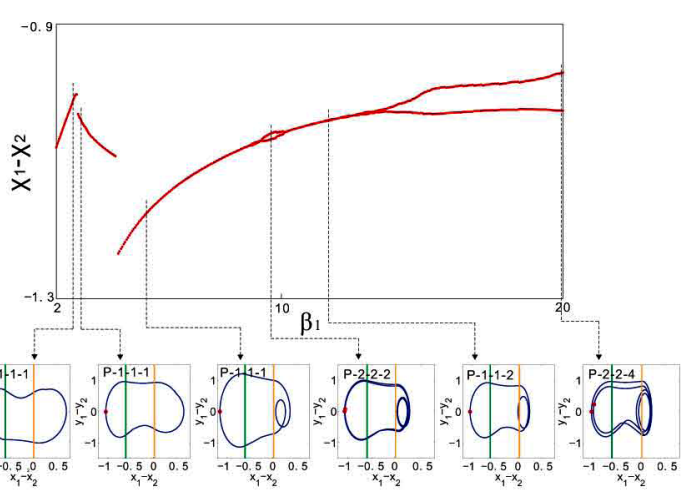

(e)

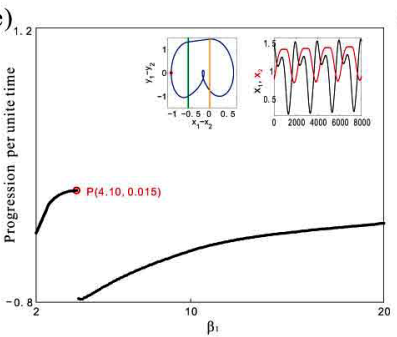

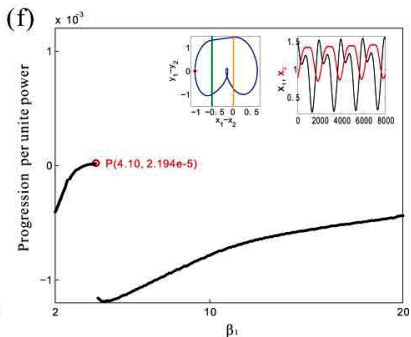

Figure 4. Bifurcation analyses of stiffness ratio for the capsule systems with one-sided (a) and double-sided (b) constraints. The nondimensional parameters are $\beta_{1} \in[2,20], \gamma=4, a=1.6, \omega=1.1, \xi=0.05, g_{1}=0.02, \beta_{2}=5, g_{2}=-0.06$. Subplots (c), (d) show the progressions per unit time and per unit power for the capsule system with one-sided constraint. Subplots (e), (f) show the progressions for the capsule system with double-sided constraint. 